\title{
Looking like a criminal: Stereotypical black facial features promote face source memory error
}

\author{
Heather M. Kleider • Sarah E. Cavrak • \\ Leslie R. Knuycky
}

Published online: 7 July 2012

(C) Psychonomic Society, Inc. 2012

\begin{abstract}
The present studies tested whether African American face type (stereotypical or nonstereotypical) facilitated stereotype-consistent categorization, and whether that categorization influenced memory accuracy and errors. Previous studies have shown that stereotypically Black features are associated with crime and violence (e.g., Blair, Judd, \& Chapleau Psychological Science 15:674-679, 2004; Blair, Judd, \& Fallman Journal of Personality and Social Psychology 87:763-778, 2004; Blair, Judd, Sadler, \& Jenkins Journal of Personality and Social Psychology 83:5-252002); here, we extended this finding to investigate whether there is a bias toward remembering and recategorizing stereotypical faces as criminals. Using category labels, consistent (or inconsistent) with race-based expectations, we tested whether face recognition and recategorization were driven by the similarity between a target's facial features and a stereotyped category (i.e., stereotypical Black faces associated with crime/violence). The results revealed that stereotypical faces were associated more often with a stereotype-consistent label (Study 1), were remembered and correctly recategorized as criminals (Studies 2-4), and were miscategorized as criminals when memory failed. These effects occurred regardless of race or gender. Together, these findings suggest that face types have strong category associations that can promote stereotype-motivated recognition errors. Implications for eyewitness accuracy are discussed.
\end{abstract}

Keywords Social cognition - Categorization · Memory · Stereotype $\cdot$ Face processing

Eyewitness memory error is the most frequent cause of wrongful convictions for prisoners who are later exonerated

H. M. Kleider $(\bowtie) \cdot$ S. E. Cavrak • L. R. Knuycky

Department of Psychology, Georgia State University,

Atlanta, GA 30030, USA

e-mail: hkleider@gsu.edu through DNA testing. False identifications are referenced in over $75 \%$ of these cases, with Black men receiving the majority of these convictions (The Innocence Project, 2008; Wells et al., 1998). Myriad studies have tested the factors that influence recognition error from a police procedural standpoint, indicating that errors are facilitated via lineup construction (Wells, 2008), other witnesses' suggestions (Clark \& Wells, 2008), and verbal face descriptions (i.e., verbal overshadowing; Schooler \& Engstler-Schooler; 1990), to name a few of these factors. Beyond police procedures, less vigorously tested have been the decision processes that underpin false identifications of Black men, who represent the largest ethnic group affected by identification errors. Although misidentifications of Black men have been linked to the cross-race effect (CRE), whereby people are better able to identify persons of their own than of other races (Meissner, Brigham, \& Butz, 2005), other previous studies have suggested that these errors may not be constrained by ingroup/out-group differences. Rather, face identification may be influenced by facial categorization that is driven by ethnic stereotypes associated with certain face types. Specifically, Black men are categorized into subgroups on the basis of the degree to which they possess stereotypically Black features (i.e., some combination of darker skin, wider nose, and fuller lips; Blair, Judd, \& Chapleau, 2004; Blair, Judd, Sadler, \& Jenkins, 2002; Eberhardt, Goff, Purdie, \& Davies, 2004). Although people are explicitly unaware of this subgrouping, these racial markers facilitate implicit face categorization, which then influences how the face is perceived and remembered (MacLin \& Malpass, 2001; Michel, Corneille, \& Rossion, 2010).

Black men retaining these stereotypical facial features are considered to represent the perception of a "prototypical" Black male. Relative to Black men with less stereotypical Black facial features (hereafter called "atypical Black features"), stereotypical facial features have been shown to 
activate automatic associations with negative behavioral stereotypes of Black men, such as aggression, violence, and criminality (Eberhardt et al., 2004). Participants presented with photographs of Black men (possessing a range of stereotypical facial features, alongside information about aggressive or nonaggressive behaviors) made predictions about probable future aggression. The men with stereotypical facial features were predicted to engage in more aggressive behaviors in the future than were their atypical counterparts. This feature-based link to aggression and crime also extends to biased judgments in actual trial outcomes, as Black defendants with stereotypical Black facial features receive more convictions, harsher sentences (Blair, Judd, \& Chapleau, 2004), and more death penalty recommendations (Eberhardt, Davies, Purdie-Vaughns, \& Johnson, 2006) than do defendants with atypical facial features. The subcategorizing of stereotypical Black faces as more violent and criminal than atypical Black faces suggests that similar subcategories may also affect face recognition. Thus, given a modicum of uncertainty, crime eyewitnesses may be more inclined to identify a person with facial features associated with the "criminal type" than to identify a person with "non-criminal-type" features. In the present study, we sought to identify a relationship between face recognition and subjective categorization of Black men due to face type, which may arguably be a factor facilitating eyewitness misidentifications.

Although abstract judgments related to racial face type have been shown to influence subjective outcomes in a variety of situations (e.g., sentencing decisions or aggression ratings), this relationship has not directly been tested as a component of face recognition. Face recognition studies have shown that semantic category information influences how faces are remembered. For example, Shepherd, Ellis, McMurran, and Davies (1978) had participants create sketches of a previously seen face after being told that the face was that of either a murderer or a lifeboat captain. The sketches were then rated by an independent group of participants on traits such as intelligence and attractiveness (without any category association). The lifeboat captain sketches were rated as more positive (i.e., more intelligent and attractive) than were the murderer sketches, suggesting that occupation category influenced facial encoding and retrieval, thus influencing the way that the face was reconstructed when sketched. In other words, the perception of the inherent characteristics of the person in the original photos influenced the way that the photo was processed, remembered, and reconstructed. The consistency of characteristic ratings from the participants sketching the photos, to the participants who only viewed and rated the photos, further suggests that categorization influences trait expectations. Klatzky, Martin, and Kane (1982) extended these findings and showed that faces may be selectively encoded on the basis of the degree to which a face represents a category label. Here, participants were presented with faces that had been prejudged as having a particular occupation (e.g., accountant or hairdresser), in conjunction with an occupation label that was consistent or inconsistent with the prejudgment. Face-occupation label congruency enhanced recognition accuracy but also increased false alarms to new (i.e., unseen) faces that were representative of the occupation label. More recently, Hills, Lewis, and Honey (2008) suggested that semantic information has the potential to guide the encoding process, whereby faces are stored within a category based on whether they match categorical expectations (e.g., stereotypes), thereby influencing face recognition. For example, and consistent with Klatzky et al.'s findings, presenting faces that were prejudged as being representative of a given occupation category (e.g., criminal or actor) with either inconsistent (e.g., criminal face type with an actor label) or consistent (e.g., criminal face type with a criminal label) primes resulted in better memory for faces that were paired with a consistent occupation label. Consistent face-label pairings also led to more false alarms for previously unseen faces. Importantly, these recognition errors only occurred when faces were primed with a label at presentation, or at presentation and test, but not at test alone. Thus, the authors argued that the effectiveness of category associations appears to be restricted to the memoryencoding process. This finding was especially informative because well-established face recognition theories (e.g., Hurlbert, 2001; Sporer, 2001; Valentine, 1991; Wang, Li, Fang, Tian, \& Liu, 2012) did not include a semantic component.

Race-based category activation has revealed similar category-congruent effects for racial stereotypes and stereotypical Black faces. Participants were faster at identifying degraded images of crime-relevant objects after seeing a stereotypical rather than an atypical Black face. Thus, activating a race-relevant concept (i.e., crime) induced attentional biases toward stereotypical faces and led to misidentifications in a subsequent face recognition task (Eberhardt et al., 2004, Exps. 4 and 5). Researchers suggested that the bidirectional priming of category and face type indicated a robust link between racial stereotypes and stereotypical Black facial features. Therefore, face recognition may also be influenced by the link between face type and stereotype priming. Sporer (2001) argued, with his ingroup/out-group model (IOM), that the attention focus during the encoding process is the primary impetus for the cross-race effect. Furthermore, "other-race" face categorization arguably leads to a surface processing strategy, where only features indicative of other-race membership are encoded, leading to a "they-all-look-alike" effect. According to the IOM, this occurs because only the individual facial features, and not the relationship and proximity 
of the features to one another, are identified. Thus, the subtleties that assist in differentiating one face from another are not stored into memory. Conversely, same-race faces are individuated with greater ease because a gestalt of the face is encoded (including both features and position), rather than the focus being on featural distinctions alone. Sporer argued that these effects are primarily motivated by one's desire to know the members of one's own group.

By adapting knowledge from face-processing theories with knowledge of how category labels affect attention and the processing of faces, in the present study we sought to investigate whether the subjective labels associated with race-based face types (i.e., Blair et al., 2002; Eberhardt et al., 2004) may facilitate miscategorizations of stereotypically Black faces into a criminal category. This association may ultimately be a factor underpinning eyewitness misidentifications of Black men. Specifically, Black men with stereotypical features may have a greater chance of being misidentified than do men with less racially distinct features, as the former are more closely associated with criminality. As with previous studies, in the present article we first verified that certain face types were associated with particular occupations or categories. Thus, in Study 1 we demonstrated that stereotypical facial features were associated with criminal labels, while atypical faces were most often associated with other category occupation labels (Hills et al., 2008). Once face type category associations had been established, we conducted three other studies to test whether stereotypical facial features were associated with criminal labels and whether people would use these associations in a face memory task.

Our main hypothesis was that stereotypical facial features would activate the criminal stereotype, such that people would use this face type as a cue to criminal-category membership. First, we expected that when stereotypical faces were presented in a criminal (i.e., stereotypeconsistent) category, they would be correctly recategorized at test into the criminal category more often than into a neutral (or stereotype-inconsistent) category. Second, when an error in categorization occurred (hereafter referred to as a miscategorization), the overwhelming tendency should be for the stereotypical faces (originally displayed in either neutral category) to be miscategorized as criminal. To complement these predictions, we further expected that atypical faces should be correctly recategorized into either neutral category more often than into the criminal category. When memory errors occurred for these faces, we anticipated that miscategorization should be evenly distributed between the two neutral categories. Furthermore, the proportions with which these correct and incorrect categorizations would occur for the criminal category should be higher for the stereotypical than for the atypical face type.

\section{Pilot study}

Method

\section{Participants}

The participants included 21 Georgia State University (GSU) undergraduate students. All of the students participated for course credit and self-identified their age (range = 18-40 years), gender (14 female, seven male), and race (nine Black, four White, eight other race).

\section{Procedure}

The participants rated a series of 77 Black faces (41 male, 36 female) on either attractiveness $(n=10)$ or stereotypicality $(n=11)$. All of the faces were taken from an online actor database and were randomly presented. The participants were instructed to use their own subjective criteria for their ratings (see Blair et al., 2002). For example, those rating the faces on stereotypicality were told, "Your task is to rate a series of faces on how stereotypically Black you find them. Your ratings should be based on your own opinions and criteria for what a stereotypically Black appearance means." The same verbiage was used for attractiveness ratings. Ratings were given on a 1-7 Likert scale $(1=$ not at all stereotypical/attractive, $7=$ very stereotypical/attractive). Next, participants completed a 20 -min distractor task. Finally, all participants were presented with ten possible occupations and rated each on its similarity to a criminal. Responses were given on a $1-6$ Likert scale $(1=\mathrm{com}$ pletely different, $6=$ completely the same). The occupations were adapted from Hills et al. (2008), and included accountant, actor, artist, businessman, doctor, lawyer, philanthropist, police officer, restaurant server, and teacher/professor. The occupations were randomly presented.

\section{Results}

\section{Face ratings}

Although a set of both male and female Black faces were prerated, only the male faces were assessed in the results that follow, as they were the focus of the subsequent study. See Study 3 for assessment of the female faces. Average attractiveness and stereotypicality ratings were calculated for each face, and then each face was categorized into a face type group (atypical, range $=2.45-4.34, n=13$; medium typical, range $=4.35-5.18, n=16$; stereotypical, range $=$ 5.19-6.18, $n=12$ ), via trichotomous split, and attractiveness groups (not attractive, range $=1.80-2.70, n=21$; attractive, range $=.80-5.00, n=20$ ), via median split. We were primarily interested in the comparison between stereotypical 
and atypical faces; therefore, a 2 (face type: stereotypical, atypical) $\times 2$ (attractiveness: attractive, not attractive) between-subjects analysis of variance (ANOVA) was conducted to test whether the stereotypical faces were more stereotypical than the atypical faces. The medium faces were not used. As expected, we found a significant main effect of face type, such that stereotypical faces $(M=5.65)$ were rated as significantly more stereotypical than atypical faces $(M=$ $3.78), F(1,21)=69.49, p<.01, \eta_{\mathrm{p}}^{2}=.77$. No other effects were significant. Figure 1 provides an example of each face type.

\section{Category ratings}

Each occupation's average similarity rating to a criminal was calculated (range: 1.5-3.17). Previous research had shown young Black men to be negatively stereotypically associated with criminality (e.g., Eberhardt et al., 2004). Here, we sought to find two roles that were the least similar to criminal-like activity, to essentially establish discrete dichotomized points along a criminality continuum. Therefore, the two roles with the lowest association (artist, $M=1.50 ;$ teacher $/$ professor, $M=1.58$ ) were selected as neutral control categories to be used in the subsequent studies.

\section{Study 1}

In Study 1, we tested whether stereotypical Black faces were automatically associated with criminality. Participants performed a speeded judgment task in which they either agreed or disagreed that a face was representative of certain category groups. All of the participants saw all face types, but category group varied by participant. We expected that if stereotypical-Black features were associated with criminality on an implicit level, then requiring participants to make quick face-category associations would reveal this association.

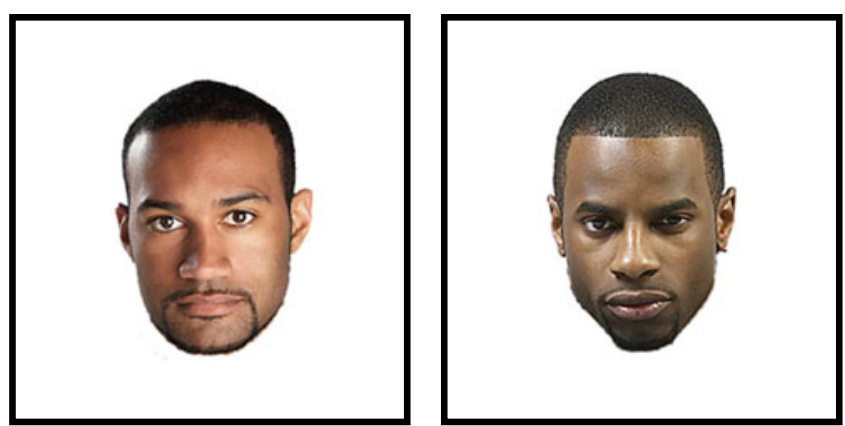

Fig. 1 Black male face type examples: atypical (left) and stereotypical (right)
Method

\section{Participants}

The participants included 30 GSU undergraduate students. The students self-identified their age (range $=18-50$ years), gender ( 22 female, 8 male), and race (15 Black, 7 White, 8 other race).

\section{Procedure}

Participants were told that they would be seeing the faces of actors who applied for a variety of movie roles, and that their job was to decide how believable the actor would be in each of the roles. Participants were randomly assigned to one role category (artist, teacher/professor, or drug dealer). A set of 12 (six atypical, six stereotypical) of the original 25 faces were randomly selected for this task (see Table 1). The category was located at the top of their computer screen, and the face was located in the middle of the screen. Faces were randomly presented, one at a time, and remained on the screen for as long as needed. The participants were told to determine whether the face represented the category "as quickly as possible," via keypress (yes/no). After all 12 faces had been viewed, the participants completed a 20-min unrelated distractor task. Finally, they completed a 28-item assessment of racial prejudice (SDS; see the Materials section below).

\section{Materials}

Social Distance Scale (Bogardus, 1925 ; Parillo \& Donoghue, 2005) The Social Distance Scale (SDS) is an established self-report measure of prejudice against African Americans wherein participants are presented with the phrase "I would be willing to have a White American person as my_ " followed by a sequence of 14 nouns (e.g., next door neighbor, romantic date). Participants rated the degree to which they agreed with each statement using a 9-point Likert scale $(1=$ strongly disagree, $9=$ strongly agree $)$. Next, participants are presented with the phrase "I would be willing to have a Black American person as my " followed by the same sequence of nouns. The averages of all responses to each set of questions (i.e., both White and Black American averages) were calculated and then subtracted from one another (White average - Black average). Higher scores reflect greater levels of prejudice against Black Americans.

Results

First, proportions were calculated depicting the number of times that a given face type was affirmatively associated 
Table 1 Mean stereotypicality and attractiveness ratings (1-7, with 7 representing the most extreme score; presented with standard deviations) for atypical and stereotypical Black male faces (Studies 1 and 2), Black female faces (Study 3), and White male faces (Study 4)

\begin{tabular}{|c|c|c|c|c|c|c|c|c|}
\hline & \multicolumn{4}{|c|}{ Stereotypicality Ratings } & \multicolumn{4}{|c|}{ Attractiveness Ratings } \\
\hline & \multicolumn{2}{|c|}{ Atypical Faces } & \multicolumn{2}{|c|}{ Stereotypical Faces } & \multicolumn{2}{|c|}{ Atypical Faces } & \multicolumn{2}{|c|}{ Stereotypical Faces } \\
\hline & $M$ & $S D$ & $M$ & $S D$ & $M$ & $S D$ & $M$ & $S D$ \\
\hline Black male faces & 3.68 & 0.64 & 5.70 & 0.41 & 3.58 & 1.02 & 2.38 & 0.53 \\
\hline Black female faces & 2.68 & 0.57 & 5.11 & 0.49 & 4.42 & 0.35 & 3.20 & 0.86 \\
\hline White male faces & 2.18 & 0.18 & 3.44 & 0.16 & 2.24 & 1.19 & 2.58 & 0.92 \\
\hline
\end{tabular}

with a particular category. That is, the number of times a participant said "yes" when a given face type was presented, divided by the total number of times a face type could be associated with a category.

Next, a 2 (face type: atypical, stereotypical) $\times 3$ (category: artist, teacher/professor, drug dealer) mixed-subjects omnibus analysis of covariance (ANCOVA), with SDS score as the covariate, ${ }^{1}$ was conducted on the proportions of affirmative category associations. A significant interaction between face type and category emerged, $F(2,26)=8.52, p<$ $.01, \eta_{\mathrm{p}}^{2}=.40$ (see Fig. 2). This interaction was decomposed by first running two univariate ANCOVAs (one for each face type). The effect for atypical faces $\left(M_{\text {artist }}=.47\right.$, $\left.M_{\text {tch } / \text { prof }}=.48, M_{\text {dealer }}=.33\right)$ was not significant, $F(2$, 26) $=.95, p=.40, \eta_{\mathrm{p}}^{2}=.07$, but the effect for stereotypical faces was significant, $F(2,26)=8.47, p<.01$, $\eta_{\mathrm{p}}^{2}=.40$. Participants were significantly more likely to associate stereotypical faces with the drug dealer category ( $M=.65)$ relative to the artist $(M=.28)$ or teacher/ professor $(M=.30)$ categories, $p \mathrm{~s}<.01$. There was no significant difference in responses between the latter categories, $p=$ .89. Next, the interaction was decomposed by running three repeated measures ANCOVAs (one for each category). The effects for the artist, $F(1,8)=3.50, p=.10, \eta_{\mathrm{p}}^{2}=.30$, and teacher/professor, $F(1,8)=3.66, p=.09, \eta_{\mathrm{p}}^{2}=.31$, categories were not significant. A significant effect only emerged for the drug dealer category, $F(1,8)=9.82, p=.01, \eta_{\mathrm{p}}^{2}=.55$, whereby participants were significantly more likely to associate the stereotypical faces than the atypical faces with the drug dealer category. Prejudice beliefs did not influence category associations: prejudice, $F(1,26)=0.21, p=.65, \eta_{\mathrm{p}}^{2}=$. 01 ; Face Type $\times$ Prejudice, $F(1,26)=0.01, p=.93, \eta_{\mathrm{p}}^{2}=.00$.

\section{Discussion}

These data support a priori expectations that stereotypically Black facial features are consistent with people's expectations

$\overline{{ }^{1} \mathrm{SDS}: M=0.01}, S D=1.13$, range $=-2.93$ to 3.21 . for criminal appearance. Moreover, asking people to make quick category judgments suggests that these stereotypical associations to criminality are automatic and culturally entrenched, as people did not have the opportunity to consider whether such associations would appear racist, and thus make more positive category associations for all faces. The only significant difference in face type and category assignment was between the stereotypical faces assigned to the drug dealer, relative to the artist and teacher/professor, category. The atypical faces were equally assigned to all categories. More to the point, the stereotypical faces were assigned more often to the drug dealer category than were the atypical faces. This effect was not the result of prejudiced attitudes (SDS) or participant race. Overall, these findings support our hypotheses that the association between stereotypical facial features and criminality is likely automatic and/or reflexive, and not reliant on one's individual perceptions of Black men as a whole.

\section{Overview of Studies 2-4}

Having established the association between criminality and face type in Study 1, we next tested whether stereotypical facial features would promote associations in memory and facilitate recognition. More specifically, stereotypical facial features may assist in remembering when a face was seen in

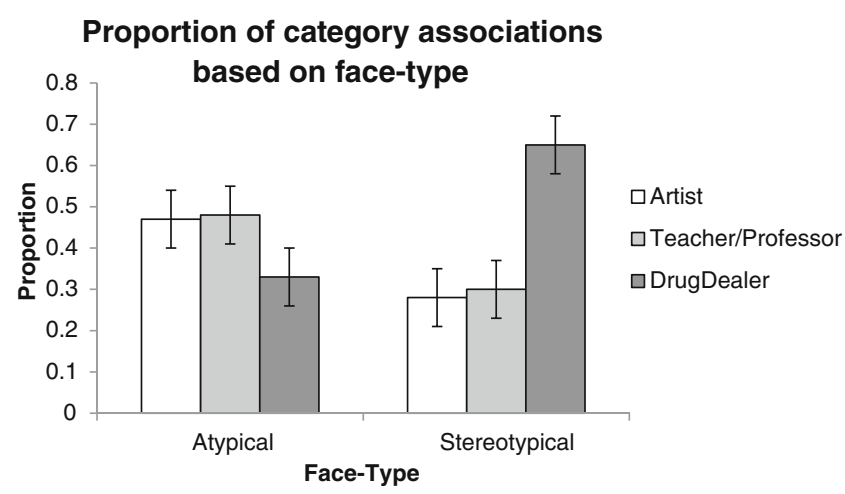

Fig. 2 Study 1: Proportions of affirmative category associations ("yes" responses) with Black male faces on the basis of face type. Error bars represent standard errors 
a category consistent with expectations (i.e., a stereotypical face presented as criminal) and promote miscategorization when a face is inconsistent with the expectations of a category (i.e., miscategorizing a stereotypical face as a criminal when it was presented as an artist or teacher/professor). This may occur (1) for Black men only as an outgrowth of the Black man-criminal stereotype (Study 2), (2) for those of an identified racial group generally (Study 3), or (3) because of face type, regardless of racial group (Study 4).

\section{Study 2}

Method

\section{Participants}

The participants included 87 GSU undergraduate students. Students self-identified their age (range $=18-30$ years), gender (65 female, 22 male), and race (50 Black, 16 White, 21 other race).

\section{Materials}

Category/face panels The 12 faces (see Table 1) used in Study 1 were randomly assigned to three category panels (four faces/panel; two atypical, two stereotypical). Each panel included a category label centered in the middle of the panel display, and the four faces were presented in two rows of two (one above the label and one below). The face positions in each panel were randomized. The categories included one stereotype-consistent label (drug dealer) and two stereotype-inconsistent (i.e., neutral) labels (artist and teacher/professor; see the pilot study).

To ensure that the relative strengths of stereotypicality did not differ across categories, a 2 (face type: atypical, stereotypical) $\times 3$ (category: artist, teacher/professor, drug dealer) between-subjects ANCOVA, with attractiveness as the covariate, was conducted on stereotypicality ratings of the faces. ${ }^{2}$ As expected, only a main effect of stereotypicality emerged, such that stereotypical faces $(M=5.48)$ were significantly more stereotypical than atypical faces $(M=3.89), F(1,5)=10.41$, $p=.02, \eta_{\mathrm{p}}^{2}=.68$. Therefore, the relative strength of face stereotypicality did not vary across panels (i.e., the stereotypical "artist" faces were not more or less stereotypical than the stereotypical "drug dealer" faces).

\section{Procedure}

The participants were told that they would see the faces of actors who had applied for a variety of movie roles, and that

\footnotetext{
${ }^{2}$ The data used here are those collected in the pilot study.
}

their job was to decide how believable and memorable they would be in each of the roles. The participants were then shown each face/category panel (see the Materials section) on the computer screen for $10 \mathrm{~s}$ (2-s ISI between panels). Then, a 20 -min distractor task was administered. Next, participants were presented with each of the original 12 faces and told to identify the role in which they had been shown at the beginning of the experiment. Each face was presented individually, and the three category options were listed below. Participants selected a category via keypress and were permitted as much time as needed to make a decision. Then they completed a racial prejudice assessment (SDS; see the Materials section of Study 1).

\section{Results}

\section{Correct recategorization}

Our first hypothesis was that face type would facilitate categorization at encoding, which would facilitate correct recategorization at test. Participants should remember and recategorize the stereotypical (vs. atypical) drug dealer faces with greater accuracy than the stereotypical faces across the other two categories. Proportions of correct recategorizations were first calculated for each Face Type $\times$ Category cell (i.e., the number of correct face recategorizations divided by the total opportunities to correctly recategorize a given face type). Then, a 2 (face type: atypical, stereotypical) $\times 3$ (category: artist, teacher/professor, drug dealer) repeated measures omnibus ANCOVA, with SDS as the covariate, ${ }^{3}$ was conducted to test the hypothesis that face type facilitates correct recategorization of faces. The results first revealed main effects for both face type, $F(1,85)=30.93, p<.01$, $\eta_{\mathrm{p}}^{2}=.27$, and category, $F(2,170)=3.28, p=.04, \eta_{\mathrm{p}}^{2}=.04$. Participants were better at recategorizing the stereotypical $(M=.39)$ than the atypical $(M=.26)$ faces, and most accurate in recategorizing the drug dealer $(M=.37)$ rather than the artist $(M=.28)$ or teacher/professor $(M=.33)$ faces. The differences were only significant when the artist and drug dealer categories were compared.

We found a significant two-way interaction between face type and category as well, $F(2,170)=14.34, p<.01, \eta_{\mathrm{p}}^{2}=.14$ (see Fig. 3). The interaction was decomposed by running a series of repeated measures ANCOVAs on each category. There were no differences between atypical and stereotypical faces across either the artist $\left[M_{\text {atyp }}=.25\right.$, $\left.M_{\text {stereo }}=.30 ; F(1,85)=1.83, p=.18, \eta_{\mathrm{p}}^{2}=.02\right]$ or teacher/professor $\left[M_{\text {atyp }}=.32, M_{\text {stereo }}=.33 ; F(1,85)=\right.$ $\left..20, p=.60, \eta_{\mathrm{p}}^{2}=.00\right]$ categories; however, consistent with our hypothesis, participants were significantly more

\footnotetext{
${ }^{3}$ SDS: $M=-0.32, S D=1.23$, range $=-5.93$ to 1.93 .
} 


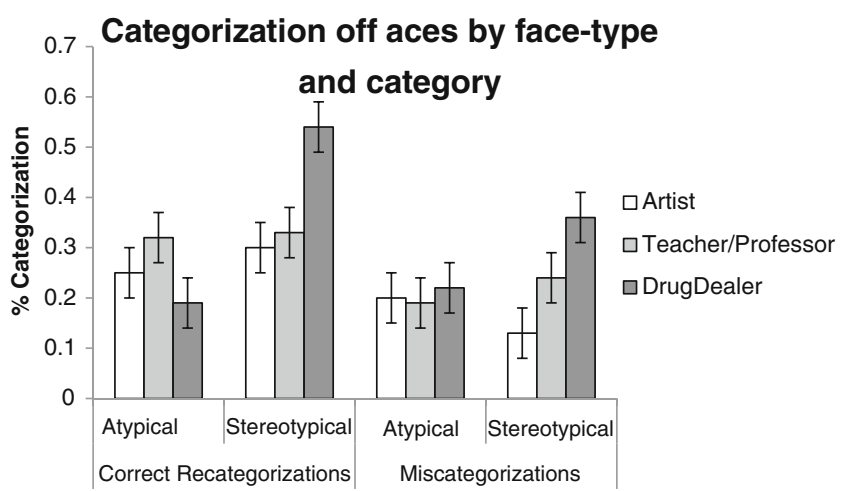

Fig. 3 Study 2: Proportions of correct recategorizations and miscategorizations of Black male faces based on face type and category label. Errors bars represent standard errors

accurate at recategorizing stereotypical $(M=.54)$ than atypical $(M=.19)$ faces, in the drug dealer category only, $F(1,85)=47.90, p<.01, \eta_{\mathrm{p}}^{2}=.36$.

\section{Miscategorization}

Our second hypothesis was that face type would facilitate miscategorization at test. When participants failed to accurately remember the original category of a face, face type should guide miscategorizations. Thus, stereotypical faces (originally presented as an artist or teacher/professor) should be miscategorized as drug dealers. Miscategorization rates were calculated for each Face Type $\times$ Category cell (i.e., number of incorrect face recategorizations divided by each participants' total incorrect recategorizations by face type). (Note: This is not simply incorrect-categorization rates, which would be the mathematical complement to the correct-recategorization rates previously reported.) Here, we are in essence determining if there was any systematic pattern in category assignments when participants' recategorizations were wrong. That is, if participants incorrectly recategorized an artist with a stereotypical face type, what did they miscategorize him as?

A 2 (face type: atypical, stereotypical) $\times 3$ (category: artist, teacher/professor, drug dealer) repeated measures omnibus ANCOVA, with SDS as the covariate, was conducted on the miscategorization rates. The results revealed main effects for both face type, $F(1,85)=10.49, p<.01, \eta_{\mathrm{p}}^{2}=.11$ , and category, $F(2,170)=11.99, p<.01, \eta_{\mathrm{p}}^{2}=.12$. Participants made significantly more miscategorizations to the stereotypical $(M=.24)$ than to atypical $(M=.20)$ faces, and significantly more miscategorizations to the drug dealer category $(M=.29)$ than to either the artist $(M=.17)$ or teacher/professor $(M=.21)$ category $(p s<.01)$. The difference between the latter two categories was statistically significant $(p=.04)$.

We found a significant two-way interaction between face type and category, as well, $F(2,170)=7.12, p<$
$.01, \eta_{\mathrm{p}}{ }^{2}=.08$ (see Fig. 3). The interaction was decomposed following procedures from the recategorization data. There were significant differences in miscategorizations to the artist, $F(1,85)=5.65, p=.02, \eta_{\mathrm{p}}^{2}=.06$, and drug dealer, $F(1,85)=12.72, p<.01, \eta_{\mathrm{p}}^{2}=.13$, categories in the predicted directions, but not to the teacher/professor category, $F(1,85)=2.73, p=.10, \eta_{\mathrm{p}}^{2}=.03\left(\right.$ artist, $M_{\mathrm{atyp}}=.20$, $M_{\text {stereo }}=.13$; drug dealer, $M_{\text {atyp }}=.22, M_{\text {stereo }}=.36$; teacher/ professor, $M_{\text {atyp }}=.19, M_{\text {stereo }}=.24$ ).

\section{Discussion}

The results support our hypotheses that stereotypical facial features are linked to criminality and serve as memory cues and/or arguably facilitate encoding of stereotypical faces into the drug dealer category. This bias resulted in correct source memory for the drug dealers, but also encouraged miscategorizations. Stereotypical faces were more accurately recategorized than atypical faces overall, but more interestingly, those faces were most often accurately recategorized as drug dealers. There were no significant differences between recategorizations of stereotypical faces into other category groups. This suggests that people used facial features as cues to category membership and this cue assisted in correct recategorization. In addition, atypical faces were more often recategorized as a teacher/professor than a drug dealer, but there were no differences in miscategorizations as a function of category presentation for this face type. Furthermore, this facial-feature cue also led people to miscategorize stereotypical faces into the drug dealer category more than into the other categories, suggesting that the association between face type and criminality was used as a default when memory failed. This suggests that this error is a general bias via face type rather than category facilitation via label.

\section{Study 3}

In previous work, Blair and colleagues (Blair, Judd, \& Fallman, 2004; Blair et al., 2002) found that participants who were asked to make qualitative judgments of personality and behavior for men of European and of African decent (with varying degrees of stereotypically Black facial features) identified target faces previously judged to be more stereotypical as also being more aggressive and criminal than faces that did not have stereotypical facial features. In the 2002 study, Blair et al. had participants rate photographs of White and Black men as to how likely they were to be the person involved in a previously read passage in which a violent or aggressive act had occurred. All photographs were independently rated for stereotypically Black facial features. Regardless of target ethnicity, Blair et al. found 
that men were most likely to be judged the protagonist in the passage when they possessed stereotypically Black features. Although the level of stereotypicality was greater for Black than for White men, the data pattern was consistent across groups. These findings were extended to archival data in criminal sentencing, in which face type, regardless of ethnicity, predicted sentence severity (Blair, Judd, \& Chapleau, 2004). Together, these studies suggest that Black facial features activate the criminal stereotype, regardless of the ethnicity of the person. However, what has not been addressed is whether this negative bias is reflective of a Black-man stereotype (regardless of the ethnicity of the target face) activated by the features themselves or more generally by a Black person who possesses these features.

The next studies focused on teasing these issues apart. In Study 3, pictures of Black women were used instead of Black men, and in Study 4, White faces with stereotypical Black features were used, testing Blair and colleagues' assumptions.

\section{Method}

\section{Participants}

Participants included 40 GSU undergraduate students. The students self-identified their age (range $=18-50$ years), gender ( 32 female, 8 male), and race ( 23 Black, 6 White, 11 other race).

\section{Materials}

Category/face panels The female faces $(n=36)$ prerated earlier (see the pilot study) were used here, and the average attractiveness and stereotypicality were calculated for each of the faces. The faces were then categorized into face-type groups (atypical, range $=1.73-3.65, n=11$; medium typical, range $=3.66-4.45, n=14$; stereotypical, range $=4.45$ $6.09, n=11)$, via trichotomous split, and attractiveness groups (not attractive, range $=2.10-3.85, n=18$; attractive, range $=3.86-5.50, n=18$ ), via median split. A 2 (face type: stereotypical, atypical) $\times 2$ (attractiveness: attractive, not attractive) between-subjects ANOVA was conducted to test whether the stereotypical faces were statistically more stereotypical than the atypical faces. Only a significant main effect of face type emerged, in that stereotypical faces $(M=$ 5.04) were rated as significantly more stereotypical than atypical faces $(M=2.95), F(1,18)=33.60, p<.01, \eta_{\mathrm{p}}^{2}=$. 65. There was no effect of attractiveness on the ratings of stereotypicality, $F(1,18)=0.14, p=.71, \eta_{\mathrm{p}}^{2}=.01$. Figure 4 provides an example of each face type.

Next, 12 faces (see Table 1) were randomly assigned to the three category panels used in Study 2 (four faces/panel;
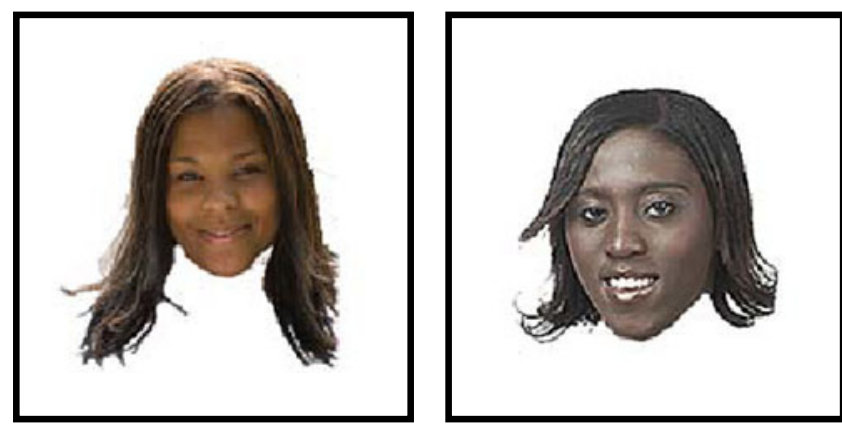

Fig. 4 Black female face type examples: atypical (left) and stereotypical (right)

two atypical, two stereotypical). The physical display of each panel was consistent with those presented in Study 2. To ensure that the relative strengths of stereotypicality did not differ across categories, a 2 (face type: atypical, stereotypical) $\times 3$ (category: artist, teacher/professor, drug dealer) between-subjects ANCOVA, with attractiveness as the covariate, was conducted on the stereotypicality ratings of faces. As expected, the only effect to emerge was a main effect of stereotypicality, such that stereotypical faces $(M=$ 5.17) were significantly more stereotypical than atypical faces $(M=2.80), F(1,5)=15.27, p=.01, \eta_{\mathrm{p}}^{2}=.75$.

\section{Procedure}

The procedure followed the protocol of Study 2 .

\section{Results}

\section{Correct recategorization}

If biased source memory is the result of general stereotypical face-type associations, we should see a pattern of results here, with the Black female faces, similar to the one that we saw with the Black male faces in Study 2. That is, stereotypical faces should be correctly recategorized as drug dealers when originally presented as drug dealers, and incorrectly miscategorized as drug dealers when originally presented in some category other than drug dealer. If, however, biased source memory is the result of a specific stereotype related to Black men and criminality only, we should not see the same data pattern that was observed in Study 2. Rather, we should see no predictable variation across correct-recategorization or miscategorization rates. Consistent with Blair, Judd, and Fallman (2004), we expected to find the facilitation of biased categorization across Black male faces only, thus fitting with the latter prediction. We should not find any effect of face type with Black female faces. 
Proportions of correct recategorizations were first calculated according to the mathematical procedure described in Study 2 . Then, a 2 (face type: atypical, stereotypical) $\times 3$ (category: artist, teacher/professor, drug dealer) repeated measures omnibus ANCOVA, with SDS as the covariate, ${ }^{4}$ was conducted on the correct-recategorization rates. Due to computer malfunctions leading to missing data, a modified sample $(n=39)$ was used for these analyses. Unexpectedly, the results first revealed a main effect of category, $F(2,74)=$ $5.93, p<.01, \eta_{\mathrm{p}}^{2}=.14$ : Participants were significantly more accurate in recategorizing the teacher/professor $(M=.39, p=$ $.04)$ and drug dealer $(M=.46, p<.01)$ faces, relative to the artist $(M=.25)$ faces. The difference between the teacher/professor and drug dealer categories was not significant $(p=.18)$.

We also found a significant two-way interaction between face type and category, $F(2,74)=25.57, p<.01, \eta_{\mathrm{p}}^{2}=.41$ (see Fig. 5). Significant differences in recategorization accuracy emerged between atypical and stereotypical faces across the teacher/professor $\left[M_{\mathrm{atyp}}=.53, M_{\text {stereo }}=.24 ; F(1,37)=20.21\right.$, $\left.p<.01, \eta_{\mathrm{p}}^{2}=.35\right]$ and drug dealer $\left[M_{\text {atyp }}=.27, M_{\text {stereo }}=.65 ; F\right.$ $\left.(1,37)=28.79, p<.01, \eta_{\mathrm{p}}^{2}=.44\right]$ categories, but not for the artist category $\left[M_{\text {atyp }}=.30, M_{\text {stereo }}=.21 ; F(1,37)=2.92, p=\right.$ $\left..10, \eta_{\mathrm{p}}^{2}=.07\right]$. Recategorization was greater for atypical (vs. stereotypical) teacher/professor faces and for stereotypical (vs. atypical) drug dealer faces.

\section{Miscategorization}

Miscategorization rates were next calculated according to the mathematical procedure described in Study 2. Then, a 2 (face type: atypical, stereotypical) $\times 3$ (category: artist, teacher/professor, drug dealer) repeated measures omnibus ANCOVA, with SDS as the covariate, was conducted on miscategorization rates. A significant two-way interaction between face type and category emerged, $F(2,74)=$ $8.79, p<.01, \eta_{\mathrm{p}}^{2}=.19$ (see Fig. 5). The effect of category was not significant for the artist $\left[M_{\text {atyp }}=.23\right.$, $\left.M_{\text {stereo }}=.14 ; F(1,37)=3.36, p=.08, \eta_{\mathrm{p}}^{2}=.08\right]$ or teacher/professor $\left[M_{\text {atyp }}=.27, M_{\text {stereo }}=.17 ; F(1,37)=\right.$ $\left.1.88, p=.18, \eta_{\mathrm{p}}^{2}=.05\right]$ categories; however there was a significant effect for the drug dealer category, such that participants miscategorized stereotypical faces $(M=.35)$ as drug dealers more than atypical faces $(M=.12), F(1,37)=$ $20.92, p<.01, \eta_{\mathrm{p}}^{2}=.36$.

\section{Discussion}

These finding suggest that biased categorization due to the Black man-criminal stereotype extends to Black women.

\footnotetext{
${ }^{4}$ SDS: $M=-0.56, S D=1.60$, range $=-4.57$ to 5.22 .
}

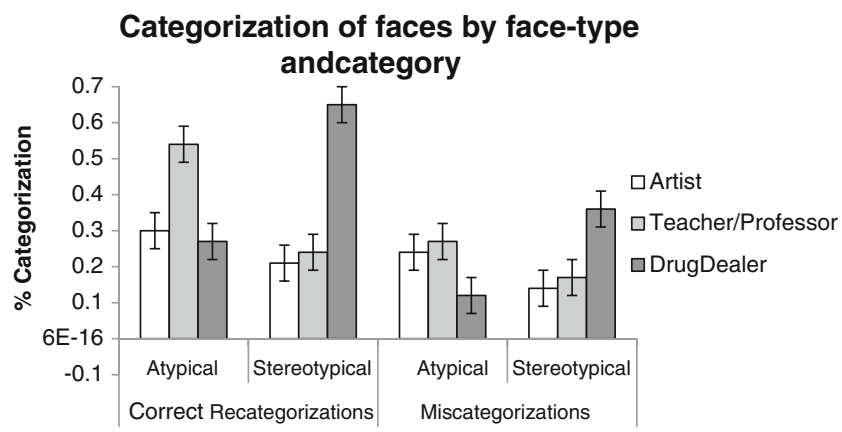

Fig. 5 Study 3: Proportions of correct recategorizations and miscategorizations of Black female faces based on face type and category label

Study 4 isolated this effect to test for a "feature effect" by using White faces with "Black" features (i.e., Blair, Judd, \& Chapleau, 2004; Eberhardt et al., 2004).

\section{Study 4}

The somewhat surprising results from Study 3 suggested that the association between criminality and Black features extends beyond Black men to include Black women. The question that remains is whether this negative association to criminality is restricted to Black facial features and Black persons, or whether it is the facial features in particular that drive the effect. In Study 4, using the same protocol as in Studies 2 and 3, we tested whether White faces with stereotypically Black features would facilitate category associations with criminal labels.

\section{Method}

\section{Participants}

The participants included 53 GSU undergraduate students. The students self-identified their age (range $=18-51$ years), gender (39 female, 12 male, 2 not identified), and race (19 Black, 19 White, 15 other race).

\section{Materials}

Category/face panels A series of White male faces $(n=54)$ taken from online sources were first prerated (see the Procedure section of the pilot study) by an independent panel of $21 \mathrm{GSU}$ undergraduates (10 participants rated the faces on attractiveness, and 11 participants provided Black stereotypicality ratings). The average attractiveness and stereotypicality scores were calculated for each face and then sorted into face-type groups (atypical, range $=2.00-2.72$, $n=17$; medium typical, range $=2.73-2.99, n=17$; or stereotypical, range $=3.00-3.64, n=20$ ), via trichotomous split, and attractiveness groups (not attractive, range $=1.11-2.33, n=28$; attractive, range $=2.34$ 
4.56, $n=26$ ), via median split. A 2 (face type: stereotypical, atypical) $\times 2$ (attractiveness: attractive, not attractive) between-subjects ANOVA was conducted. Only a main effect of face type emerged, as stereotypical faces $(M=3.24)$ were rated as significantly more stereotypical than atypical faces $(M=2.35), F(1,33)=185.12, p<.01, \eta_{\mathrm{p}}^{2}=.85$. Figure 6 provides an example of each face type.

Next, 12 faces (see Table 1) were randomly assigned to the three category panels used in Studies 2 and 3 (four faces/ panel; two atypical, two stereotypical). The presentation of the faces within each panel was identical to that reported in Studies 2 and 3. A 2 (face type: atypical, stereotypical) $\times 3$ (category: artist, teacher/professor, drug dealer) betweensubjects ANCOVA, with attractiveness as the covariate, was conducted to ensure that the relative strengths of stereotypicality did not differ across categories. As expected, the only effect to emerge was a main effect of stereotypicality, $M_{\text {stereo }}=$ $3.43, M_{\text {atyp }}=2.19, F(1,5)=80.50, p<.01, \eta_{\mathrm{p}}^{2}=.94$.

\section{Procedure}

The procedure followed the protocol of Studies 2 and 3.

Results

\section{Correct recategorization}

Consistent with Blair et al. (2002; see also Blair, Judd, \& Chapleau, 2004; Blair, Judd, \& Fallman, 2004), if biased categorization is the result of general stereotypical face-type associations, we should see a pattern of results here similar to the ones that were observed with both the Black male and female faces in Studies 2 and 3. That is, White faces with stereotypically Black features should be correctly recategorized as drug dealers when originally presented as drug dealers, and incorrectly miscategorized as drug dealers when originally presented as some category other than drug dealer. If, however, biased categorization is the result of a specific stereotype related to Black persons only, we should not see the same data pattern that was observed in the previous studies. Rather, we should see no predictable variation across correctrecategorization or miscategorization rates.

Proportions of correct recategorizations were calculated according to the procedure outlined in Study 2. Then, a 2 (face type: atypical, stereotypical) $\times 3$ (category: artist, teacher/professor, drug dealer) repeated measures omnibus ANCOVA, with SDS as the covariate, ${ }^{5}$ was conducted on recategorization rates. Due to computer malfunctions leading to missing data, a modified sample $(n=48)$ was used for

$\overline{{ }^{5} \text { SDS: } M=0.26}, S D=1.41$, range $=-2.64$ to 6.14 .
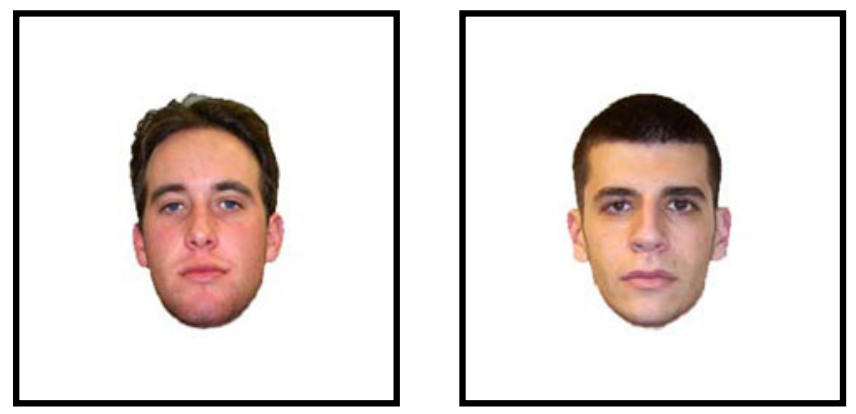

Fig. 6 White male face type examples: atypical (left) and stereotypical (right)

these analyses. The results first revealed a main effect of category, $F(2,92)=12.34, p<.01, \eta_{\mathrm{p}}^{2}=.21$. Consistent with Study 3 , participants were significantly more accurate in recategorizing the teacher/professor $(M=.35)$ and drug dealer $(M=.34)$ faces, relative to the artist $(M=.17)$ faces, $p \mathrm{~s}<.01$, but the difference between the teacher/professor and drug dealer faces was not significant, $p=.82$.

We also found a significant two-way interaction between face type and category, $F(2,92)=3.59, p=.03, \eta_{\mathrm{p}}^{2}=.07$ (see Fig. 7). The effect of face type was only significant for the drug dealer category $\left[M_{\text {atyp }}=.26, M_{\text {stereo }}=.43 ; F(1,46)=\right.$ $\left.5.94, p=.02, \eta_{\mathrm{p}}{ }^{2}=.11\right]$, but not for either the artist $\left[M_{\text {atyp }}=\right.$ $\left..23, M_{\text {stereo }}=.11 ; F(1,46)=3.28, p=.08, \eta_{\mathrm{p}}^{2}=.07\right]$ or the teacher/professor $\left[M_{\text {atyp }}=.38, M_{\text {stereo }}=.33 ; F(1,46)=.12, p=\right.$ $\left..75, \eta_{\mathrm{p}}^{2}=.00\right]$ category. Consistent with Studies 2 and 3 , stereotypical drug dealer faces were recategorized better than atypical drug dealer faces.

\section{Miscategorization}

Miscategorization rates were calculated according to the procedure outlined in Study 2. Then, a 2 (face type: atypical, stereotypical) $\times 3$ (category: artist, teacher/professor, drug dealer) repeated measures omnibus ANCOVA, with SDS as

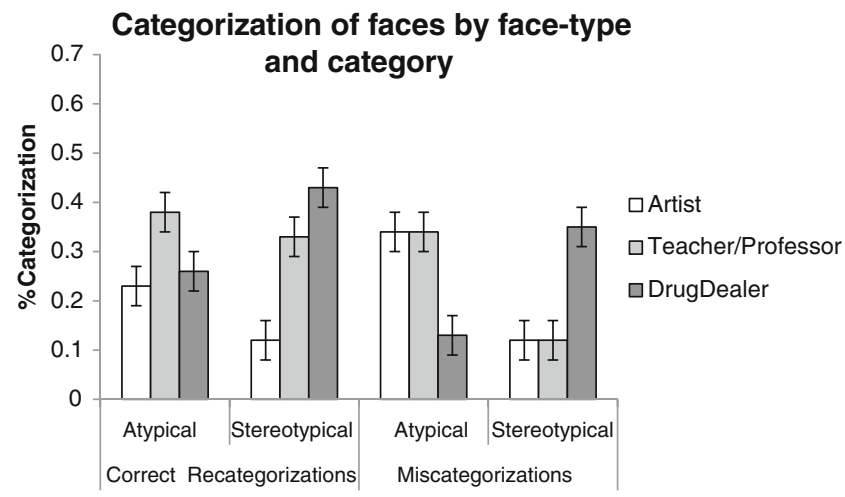

Fig. 7 Study 4: Proportions of correct recategorizations and miscategorizations of White male faces based on face type and category label. Error bars represent standard errors 
the covariate, was conducted. A modified sample $(n=47)$ was again used here. A significant main effect of face type emerged, $F(1,45)=15.95, p<.01, \eta_{\mathrm{p}}{ }^{2}=.26$, such that atypical faces $(M=.27)$ were miscategorized more often than stereotypical faces $(M=.20)$. This is in opposition to the findings in Study 2 with Black target faces, wherein the stereotypical faces were more often miscategorized. However, this did not influence the expected overall outcome, but may have indicated that people did not attend to White faces with expected features at the same level that they attended to White faces with "Black" features, and thus did not remember them as well.

A two-way interaction between face type and category also emerged, $F(2,90)=35.26, p<.01, \eta_{\mathrm{p}}{ }^{2}=.44$. The effect of face type was significant across all categories: artist, $F(1,45)=$ $46.21, p<.01, \eta_{\mathrm{p}}^{2}=.51$; teacher/professor, $F(1,45)=$ 28.92, $p<.01, \eta_{\mathrm{p}}^{2}=.39$; drug dealer, $F(1,45)=26.60, p<$ $.01, \eta_{\mathrm{p}}^{2}=.37$. Participants miscategorized atypical faces as artists or teacher/professors more often than stereotypical faces $\left(M_{\text {artist }}=.34, M_{\text {tchr } / \text { prof }}=.34, M_{\text {dealer }}=.13\right)$ and miscategorized stereotypical faces as drug dealers more often than atypical faces $\left(M_{\text {artist }}=.12, M_{\text {tch } / \text { prof }}=.12, M_{\text {dealer }}=.35\right)$.

\section{Discussion}

The findings in Studies 3 and 4 showed that people made the same biased judgments, correct as well as incorrect category assignments to the drug dealer category, for Black female faces and White male faces with stereotypically Black features. This finding is consistent with earlier work by Blair and colleagues (2002; Blair, Judd, \& Chapleau, 2004) demonstrating that the criminality bias is deeply rooted in facial features that cue the association between Black people and violence/aggression, regardless of whether the target face is actually Black.

\section{General discussion}

The findings in these studies revealed that, for both Black and non-Black participants, face recognition was influenced by associations between criminal category labels and highly ethnic facial features (i.e., stereotypical faces). In Study 1, participants associated stereotypically Black faces with a criminal category (drug dealer) more often than with neutral categories (artist, teacher/professor). In Studies 2 and 3, stereotypically Black (male and female) faces were correctly categorized as drug dealers more often than other category labels when they were originally presented as such. These faces were also more likely to be miscategorized as drug dealers when they were originally presented within another category. Moreover, atypical faces were associated most with neutral labels. Finally, in Study 4, White male faces, prejudged for stereotypical Black features, were also more often accurately and inaccurately categorized as drug dealers than were atypical-featured faces. This finding suggests that the association between criminality and ethnically Black facial features extends to anyone who possesses these features, regardless of whether they are Black. Potentially, these biased associations between criminality and stereotypical face type may underpin the misidentifications that plague the court system and are especially prevalent for Black men.

Several studies have demonstrated that racial category membership is a flexible construct. For example, MacLin and Malpass (2001) found that faces with racially ambiguous features were more likely to be categorized as either Black or Hispanic on the basis of one distinct racial marker (e.g., hair style) and that these faces were subsequently misrecognized. The authors suggested that racial markers can cause a face to be perceived and remembered differently. Top-down factors such as activating racial identity within an observer also influences face categorization. Chiao, Heck, Nakayama, and Ambady (2006) varied the racial identity priming of Black and White biracial participants prior to their performing a visual search task for Black or White faces. The results showed that the search pattern was significantly different for participants primed with their White rather than their Black identity, suggesting that flexibility in face processing may be influenced by subjective context. Similarly, in the present study, subjective inferences of racial category membership (i.e., stronger links to Black ethnicity as a function of face type) influenced face processing and categorization. Moreover, the present study required not only face recognition but tying the particular faces to a given category or source of familiarity. Thus, to correctly recategorize target faces, participants had to recruit cues to source as well as to familiarity information from memory. When memorial information is ambiguous and source information is difficult to retrieve, people rely on heuristic expectations to assist them (Kleider, Pezdek, Goldinger, \& Kirk, 2008). In this study, those expectations included stereotypic information associated with face types and criminal behavior. Similar mental shortcuts to processing are employed to explain the cross-race effect.

The Sporer (2001) model of cross-race face recognition suggests that categorization of faces as in-group or outgroup members occurs early in the face recognition process, and thus guides the quality of facial details that are stored with the face, which then dictates accurate face recognition. He suggested that the categorization works as a faceprocessing shortcut, so that in-group faces are given more attention, and potentially cognitive space, than are outgroup faces (see also Levin, 1996; MacLin \& Malpass, 2001, 2003). Applying this idea to the present study, we 
suggest that stereotypical faces activate expectations of criminality, and thus are readily associated with the drug dealer category. Once a face is categorized as criminal, processing of specific facial features ceases or is given fewer processing resources during encoding, which makes the face difficult to differentiate from other faces at retrieval. That is, in the present study, all of the stereotypical faces that are stored as criminals will be similarly activated at retrieval, setting the stage for misidentification. This explanation is also supported by false-memory models, which suggest that event information is stored both with specific (i.e., verbatim) and schematic (i.e., gist) memory traces. Thus, during retrieval, specific and schematic forms of information are retrieved, and depending on the cues presented at retrieval, the gist-based information may afford the strongest response and the bulk of the remembered information (Brainerd \& Reyna, 2002; Reyna \& Brainerd, 1995). The findings in the present study demonstrate a similar gist-based retrieval instantiation, as the face recognition errors seem to be based on a schematic expectation for criminal behavior linked to a certain face type. If the retrieval was verbatim-trace activation, the miscategorization errors of stereotypical face types into the drug dealer category would not occur at a rate greater than chance.

The findings of this study also fit nicely within established face recognition theories, such as the multidimensional space model (MDS model; Valentine, 1991). The argument here is that all dimensions of a face are clustered around a central tendency in face space. Faces that are atypical are easily recognized, because the location where these faces are theoretically stored is less populated with exemplars than the one where typical faces are stored. Also, atypical faces are more rapidly retrieved than typical faces, which require more filtering to retrieve the target face. One could argue that the stereotypically Black faces are more typical of the Black man category than are atypical faces, and thus are stored more closely together. This density makes for more false alarms for these typical faces. To build upon this theory, researchers have suggested that facial features may constitute their own dimension or that race, gender, and age may also be processed individually (Rhodes, 1988). However, this is still a debated topic. The findings of this study suggest a semantic component related to race that has not been included in previous face recognition models.

Myriad studies in the social psychology literature have demonstrated biased evaluations of Black men as criminals (e.g., Eberhardt et al., 2004; Pizzi, Blair, \& Judd, 2005). A review of courtroom decisions suggests that Black men are judged harshly, implying that people (both jurors and judges) hold stereotypic expectations toward Black men (Sommers \& Ellsworth, 2001). This negative association may arguably be rooted in cultural mores from both the past and current media coverage of Black men and criminal behavior (e.g., Sommers, Apfelbaum, Dukes, Toosi, \& Wang, 2006). Littlefield (2008) suggested that the use of media to portray a stereotyped view has been used in reference to Black women, as well.

Building on this previous work by Eberhardt (e.g., Eberhardt et al., 2004) and others, we expected that face type would facilitate criminal recategorization. We also expected that this semantic categorization would motivate both correct categorization and miscategorization of Black men who possessed stereotypical features. Therefore, the findings in Studies 1 and 2 were consistent with previous work and supported our main hypothesis that Black men with stereotypical features were more likely to be misidentified as criminals than were men with atypical features. We considered that on the ethnic-identity continuum, where faces with stereotypical Black features were most representative of Black ethnicity (and all negative associations), and atypical faces were on the opposite end of the continuum (and the most "White-like" of the Black faces), they were most likely to be associated with positive traits. This expectation was supported in all of our studies. The faces with atypical features were most often correctly categorized and miscategorized into neutral categories, suggesting that these faces were the least representative of the Black prototype, and thus were associated with relatively more positive traits.

We expected the negative association of criminality and face type to be restricted to Black men; however, surprisingly, it was not. The majority of crime stories in the media depict Black men in the criminal role. In addition, the Black man-criminal stereotype has not been discussed in the literature as a Black "person" stereotype. Thus, the findings of Study 3, which revealed a data pattern similar to Study 2, were unexpected. The results showed the same bias with categorization of stereotypically featured Black women that had been demonstrated with Black men: correct categorization and miscategorization of those faces into the drug dealer category. Moreover, the atypical faces were also more often correctly categorized and miscategorized as belonging to neutral categories rather than to the drug dealer category. This suggests that the negative association of Black features and criminality is also in force for Black women, and supports a recent study by Viglione, Hannon, and DeFina (2011) that demonstrated that Black women with lighter skin received more lenient prison sentences and served less time in prison overall than did Black women with relatively darker skin complexions. This finding opened a hole in our theory about criminality and Black men's prototypical features. Is it possible that the features alone, rather than a person's "Blackness," drives this association?

Motivated by previous work by Blair, Judd, and Chapleau (2004), who found that White faces could have Black-like features, Study 4 was conducted using White 
men's faces that were prejudged for stereotypically Black features. As was found in the previous studies, White men with stereotypically Black features were categorized and miscategorized into the drug dealer category more than into the other categories. In addition, the atypical White men were categorized and miscategorized into the neutral categories most often.

Together, these findings suggest that the stereotypical features that a face possesses are what drive the categorization into the criminal category, and not the demographics of the person, per se. Thus, the association between criminality and Afrocentric features is universal, regardless of gender or race. The present article is the first to empirically demonstrate this broader connection. Moreover, and consistent with the social psychology literature (e.g., Blair, Judd, \& Fallman, 2004), these data suggest that the negative bias and judgments that are associated with being stereotypically Black occur automatically and are likely based on longheld cultural and societal beliefs that Black is negative, and even less than human (Goff, Eberhardt, Williams, \& Jackson, 2008). Notably, these findings were consistent across all of our studies, regardless of level of prejudice attitude or participant ethnicity, which points to the roots of this biased association being prevalent in society as a whole rather than just within a subgroup of individuals who hold certain beliefs or are exposed to certain groups of people.

One possible weakness of this study is that we used only drug dealer as a criminal category. This label was intentionally selected, as it did not necessarily suggest violence, as other category labels would (e.g., murderer, rapist), but did suggest antisocial behavior. Therefore, it may be that stereotypical face types are not associated with "all" criminal activity, but only with certain levels or types of criminal activity. This question may be investigated in future studies. What was established here is that people associated stereotypical facial features with the drug dealer category, which is clearly a negative category. This association assisted in the categorization of stereotypical faces, but led to miscategorizations as well.

Author note Heather M. Kleider, Ph.D., Department of Psychology, Georgia State University.

Correspondence concerning this article should be addressed to Heather M. Kleider, Department of Psychology, Georgia State University, Atlanta, GA 30030. Telephone: 404-413-6280. Email: hkleider@gsu.edu

\section{References}

Blair, I. V., Judd, C. M., \& Chapleau, K. M. (2004a). The influence of Afrocentric facial features in criminal sentencing. Psychological Science, 15, 674-679. doi:10.1111/j.0956-7976.2004.00739.x

Blair, I. V., Judd, C. M., \& Fallman, J. L. (2004b). The automaticity of race and Afrocentric facial features in social judgments. Journal of Personality and Social Psychology, 87, 763-778. doi:10.1037/ 0022-3514.87.6.763

Blair, I. V., Judd, C. M., Sadler, M. S., \& Jenkins, C. (2002). The role of Afrocentric features in person perception: Judging by features and categories. Journal of Personality and Social Psychology, 83, 5-25. doi:10.1037/0022-3514.83.1.5

Bogardus, E. S. (1925). Measuring social distance. Journal of Applied Sociology, 9, 299-309.

Brainerd, C. J., \& Reyna, V. F. (2002). Fuzzy-trace theory and false memory. Current Directions in Psychological Science, 11, 164169. doi:10.1111/1467-8721.00192

Chiao, J. Y., Heck, H. E., Nakayama, K., \& Ambady, N. (2006). Priming race in biracial observers affects visual search for Black and White faces. Psychological Science, 17, 387-392. doi:10.1111/j.1467-9280.2006.01717.x

Clark, S. E., \& Wells, G. L. (2008). On the diagnosticity of multiplewitness identifications. Law and Human Behavior, 32, 406-422. doi:10.1007/s10979-007-9115-7

Eberhardt, J. L., Davies, P. G., Purdie-Vaughns, V. J., \& Johnson, S. (2006). Looking deathworthy: Perceived stereotypicality of Black defendants predicts capital-sentencing outcomes. Psychological Science, 17, 383-386. doi:10.1111/j.1467-9280.2006.01716.x

Eberhardt, J. L., Goff, P., Purdie, V. J., \& Davies, P. G. (2004). Seeing Black: Race, crime, and visual processing. Journal of Personality and Social Psychology, 87, 876-893. doi:10.1037/00223514.87.6.876

Goff, P. A., Eberhardt, J. L., Williams, M. J., \& Jackson, M. (2008). Not yet human: Implicit knowledge, historical dehumanization, and contemporary consequences. Journal of Personality and Social Psychology, 94, 292-306. doi:10.1037/0022-3514.94.2.292

Hills, P. J., Lewis, M. B., \& Honey, R. C. (2008). Stereotype priming in face recognition: Interactions between semantic and visual information in face encoding. Cognition, 108, 185-200. doi:10.1016/ j.cognition.2008.03.004

Hurlbert, A. (2001). Trading faces. Nature Neuroscience, 4, 3-5. doi: $10.1038 / 82877$

Klatzky, R. L., Martin, G. L., \& Kane, R. A. (1982). Semantic interpretation effects on memory for faces. Memory \& Cognition, 10, 195-206. doi:10.3758/BF03197630

Kleider, H. M., Pezdek, K., Goldinger, S. D., \& Kirk, A. (2008). Schema-driven source misattribution errors: Remembering the expected from a witnessed event. Applied Cognitive Psychology, 22, 1-20. doi:10.1002/acp.1361

Levin, D. T. (1996). Classifying faces by race: The structure of face categories. Journal of Experimental Psychology: Learning, Memory, and Cognition, 22, 1364-1382. doi:10.1037/02787393.22.6.1364

Littlefield, M. (2008). The media as a system of racialization: Exploring images of African American women and the new racism. American Behavioral Scientist, 51, 675-685. doi:10.1177/ 0002764207307747

MacLin, O. H., \& Malpass, R. S. (2001). Racial categorization of faces: The ambiguous race face effect. Psychology, Public Policy, and Law, 7, 98-118. doi:10.1037/1076-8971.7.1.98

MacLin, O. H., \& Malpass, R. S. (2003). The ambiguous-race face illusion. Perception, 32, 249-252. doi:10.1068/p5046

Meissner, C. A., Brigham, J. C., \& Butz, D. A. (2005). Memory for own- and other-race faces: A dual-process approach. Applied Cognitive Psychology, 19, 545-567. doi:10.1002/acp.1097

Michel, C., Corneille, O., \& Rossion, B. (2010). Holistic face encoding is modulated by perceived face race: Evidence from perceptual adaptation. Visual Cognition, 18, 434-455. doi:10.1080/ 13506280902819697

Parrillo, V. N., \& Donoghue, C. (2005). Updating the Bogardus Social Distance Studies: A new national survey. The Social Science Journal, 42, 257-271. 
Pizzi, W. T., Blair, I. V., \& Judd, C. M. (2005). Discrimination in sentencing on the basis of Afrocentric features. Michigan Journal of Race \& Law, 10, 1-27.

Reyna, V. F., \& Brainerd, C. J. (1995). Fuzzy-trace theory: An interim synthesis. Learning and Individual Differences, 7, 1-75. doi:10.1016/1041-6080(95)90031-4

Rhodes, G. (1988). Looking at faces: First-order and second-order features as determinants of facial appearance. Perception, 17, 43-63. doi:10.1068/p170043

Schooler, J. W., \& Engstler-Schooler, T. Y. (1990). Verbal overshadowing of visual memories: Some things are better left unsaid. Cognitive Psychology, 22, 36-71. doi:10.1016/0010-0285(90)90003-M

Shepherd, J. W., Ellis, H. D., McMurran, M., \& Davies, G. M. (1978). Effect of character attribution on Photofit construction of a face. European Journal of Social Psychology, 8, 263-268. doi:10.1002/ejsp.2420080210

Sommers, S. R., Apfelbaum, E. P., Dukes, K. N., Toosi, N., \& Wang, E. J. (2006). Race and media coverage of hurricane katrina: Analysis, implications, and future research questions. Analyses of Social Issues and Public Policy, 6, 39-55. doi:10.1111/j.1530-2415.2006.00103.x

Sommers, S. R., \& Ellsworth, P. C. (2001). White juror bias: An investigation of prejudice against Black defendants in the American courtroom. Psychology, Public Policy, and Law, 7, 201-229. doi:10.1037/1076-8971.7.1.201

Sporer, S. L. (2001). Recognizing faces of other ethnic groups: An integration of theories. Psychology, Public Policy, and Law, 7, 36-97. doi:10.1037/1076-8971.7.1.36

Valentine, T. (1991). A unified account of the effects of distinctiveness, inversion, and race in face recognition. Quarterly Journal of Experimental Psychology, 43A, 161-204. doi:10.1080/14640749108400966

Viglione, J., Hannon, L., \& DeFina, R. (2011). The impact of light skin on prison time for Black female offenders. The Social Science Journal, 48, 250-258. doi:10.1016/j.soscij.2010.08.003

Wang, R., Li, J., Fang, H., Tian, M., \& Liu, J. (2012). Individual differences in holistic processing predict face recognition ability. Psychological Science, 23, 169-177. doi:10.1177/0956797611420575

Wells, G. L. (2008). Field experiments on eyewitness identification: Towards a better understanding of pitfalls and prospects. Law and Human Behavior, 32, 6-10. doi:10.1007/s10979-007-9098-4

Wells, G. L., Small, M., Penrod, S., Malpass, R. S., Fulero, S. M., \& Brimacombe, C. E. (1998). Eyewitness identification procedures: Recommendations for lineups and photospreads. Law and Human Behavior, 22, 603-647. doi:10.1023/A:1025750605807 